\title{
Have You Seen A Kaleidoscope? A Case of Visual Migraine Aura
}

\section{Fatimah Lateef}

Senior Consultant

Department of Emergency Medicine, Singapore General Hospital

Professor, Duke NUS Graduate Medical School

Professor, Yong Loo Lin School of Medicine, National University of Singapore

Professor, Lee Kong Chian Medical School, Nanyang Technological University

Director, Sing Health Duke NUS Institute of Medical Simulation (SIMS)

*Corresponding Author: Fatimah Lateef, Dept of Emergency Medicine, Singapore General Hospital.

Received date: November 19, 2021; Accepted date: December 27, 2021; Published date: January 05, 2022

Citation: Fatimah Lateef (2022). Evisceration Secondary to Coughing in a Child Undergoing Ventriculoperitoneal Shunt Surgery. J. Archives of Medical Case Reports and Case Study, 5(1); DOI:10.31579/2692-9392/095

Copyright: (C) 2022 Fatimah Lateef, This is an open access article distributed under the Creative Commons Attribution License, which permits unrestricted use, distribution, and reproduction in any medium, provided the original work is properly cited.

\begin{abstract}
This is a case of migraine, presenting with the interesting visual aura of a 'kaleidoscope': waves with flickering movements made up of a variety of colors (red, blue, green, yellow), commencing laterally and spreading superiorly in the visual field of the left eye. This description is quite similar to a fortification spectrum, which usually expands and spreads in a C-shape fashion over one side of the visual field.

Several different possible pathophysiological explanation are shared as well as the common differential diagnoses. In the work up of a patient with visual aura migraine, a comprehensive and detailed history is important, together with the appropriate investigations, in order not to miss any serious syndromes, including stroke syndromes and epilepsy.

Key words: 'kaleidoscopic' visual aura; migraine; fortification spectrum
\end{abstract}

\section{The Case}

A 54 year old lady with a history of migraine for many years (where she only has headaches), presented with new onset visual symptom. There was narrowing of the visual field in her left eye, whereby the area affected was the outer and lateral part of the visual field. At the same time, she noticed wave-like, flickering movements comprising of variety of colors (red, blue, green and yellow), commencing laterally and slowly spreading to the superior part of the visual field in her left eye. She described this as an appearance she had seen through a 'kaleidoscope'. There were no associated scotomas or sudden blindness and she was still able to identify normal images whilst experiencing the attack. She has observed this only in the last 6 months, with the attack coming on about once a month. Each episode would last about 15-20 minutes. The visual symptom started first and is followed by the headache. By the time the headache comes on, the visual symptoms would have subsided. The headache may last anything between 30 minutes to several hours. There was one occasion when she developed vertiginous giddiness after the visual aura symptom. There were no sensory or motor disturbances associated with these attacks. Also, the attack has only happened in her left eye and never in the right eye, thus far. The patient has never been on any regular drugs nor medications. For her migraine, she takes a combination of paracetamol, diclofenac and prochloperazine as needed. The only significant family history is her mum who has a history of migraine, without any visual aura.

At presentation, the examination of the patient was completely normal: stable vital signs, no fever, no localizing cranial nerves or neurological signs. Her blood investigations, full blood count, electrolytes, renal function, thyroid function test, liver function test and calciummagnesium-phosphate were all normal. Her ESR (erythrocyte sedimentation rate), protein $\mathrm{S}$, protein $\mathrm{C}$, anti-thrombin III factor and antiphospholipid antibody were all normal. The CT scan and Magnetic Resonance Imaging (MRI) of the brain (done after the attack) were also normal. An EEG (electroencephalography) done was also normal.

A final diagnosis of Migraine with Visual Aura was made.

\section{Migraine with Visual Aura}

Migraine is a common headache presentation, of varying severity and nature. It is more common in women and can occur either without or with aura, which are usually completely reversible. Migraine triggers are quite common and is reported by some $80 \%$ of migraine patients. Change in 
weather, intake of certain types of food, lack of sleep, certain drug and trauma can be triggers. Aura symptoms may manifest either as sensory, visual or language (or aphasia) disturbances. [1-3] Visual auras are usually the commonest and the most multifaceted. A wide spectrum and range of visual manifestations have been reported in the literature (Table 1). [3-5, 7-10]

\begin{tabular}{|l|l|}
\hline Table 1: & Range of Visual Aura Symptoms Associated with Migraine (3-5, 7-10) \\
\hline & -Elementary visual disturbances eg. zig zag lines, crescent shapes, flickering lights \\
& -Flashes of bright lights \\
-Blurred vision & -Scotomas, scintillating scotomas (single or multiple blind area/s) \\
-Small bright dots & -Deformed images (alteration of outlines and angles) \\
-Tunnel vision (blindness in the whole periphery) & -Black spots or white spots \\
-Blind spots & -Alteration in the perception of distance \\
-"Mosaic" or fractured vision (seeing like a mosaic or broken up images) & -Complex hallucinations( visual perception of things not present) \\
-Corona phenomenon (seeing an extra edge on objects) \\
-"Negative film" ( seeing like a negative of a film) \\
-Macropsia or micropsia ( things look larger or smaller than they really are) \\
-Hemianopsia (blindness of half the visual field) \\
-Things looking closer than they really are \\
-Things looking further than they really are \\
-Visual snow (Dynamic and continuous black/ grey dots on white background or \\
white/ grey dots on black background)
\end{tabular}

Our patient would be classified as having migraine with aura. [1] Such patients can still have a migraine attack without aura as well. The patient's visual symptoms with flickering, bright and multi-colored waves of lights appear to fit into the description of a 'fortification spectrum' that usually expands in a C-shape over one side (lateral aspect) of the visual field, only in her left eye, thus far. The gradual spread that she described is one of the more typical feature of a migraine aura. These visual symptoms are fully reversible. The duration of her aura lasted less than an hour, which is the usual. If the aura lasted more than 60 minutes, it would be classified as a persistent aura without infarction. [3,4, 7-9]

Visual aura usually last between 10-30 minutes. Kaleidoscopic hallucinations are thought to originate in the prestriate area of the brain whilst the zigzag patterns of teichopsia is generated in the striate cortex. This usually is seen in the peripheral vision as wavy images or even dazzling fragments. The brain creates the visual hallucination of 'fractured' or bright lights similar to the image when looking through a kaleidoscope. Others have described it as "dancing grains of light". This is often a short-lived distortion of image and as in our patient, only lasted a few minutes. Kaleidoscopic hallucination as this can affect either one or both eyes, but usually unequally. It commences from the side (lateral aspect of the visual field) and 'marches' inwards. The headache may come on with the visual aura or after it has subsided. [3, 10-12]

\section{Differential Diagnoses and Pathophysiology}

For a patient presenting with visual aura migraine such as the kaleidoscopic visual aura, for the first time, a work up will be necessary. Transient ischaemic attacks, stroke or a cerebro-vascular event would have to be ruled out. It can also be associated with serious brain injury in a trauma patient. Sometimes this can also be a first presentation for multiple sclerosis (MS). In the approach to the patient, the possibility of retinal migraine (ocular migraine) must be considered. Ocular migraine causes brief attacks of visual symptoms such as flashing, which are usually short-lived and not associated with organic causes. In the rare case of ocular migraine, there may be associated reduced blood flow, which may have some consequence on the retina blood supply. It is thought to be associated with smoking, hypertension, hypoglycaemia or stress. Besides this, ocular migraine can present with scotomas and transient loss of vision. Epilepsy would also have to be ruled out and performing an EEG (electroencephalography) is part of the work up as well. In fact, there is a term, migralepsy, which has been coined and is used to describe a seizure occuring during or within 1 hour of a typical migraine with aura attack. $[6,11,13-15]$

The pathophysiology of the visual auras has been said to be due to one or a combination of the following: [8-10, 16-25]

1. Commencement of a wave of neuronal hyper-activity followed by a wave of hypo-activity, spreading across the brain lobes. The rate of spread is estimated to be about $3 \mathrm{~mm}$ per minute. This has also been described as a central spreading depression or 'cortical spreading depolarization' (CSD), which has been observed by researchers from functional MRI, in some patients with migraine. To date, not much is known about possible biomarkers of CSD that might predispose migraine sufferers to aura. [10, 12, 19, 20, 22]

2. Electro-cortical activities or phenomenon, called the " $\mathrm{H}$ " response, to flicker stimulation in EEG (electroencephalography) studies of migraine patients. These have been noted to give a significantly greater activity than in the control groups of patients without migraine. The activity is noted predominantly in the temporo-prietal region in some cases. Analysis of spontaneous EEG activities in some cases also showed increased alpha wave rhythm response over the posterior regions of the brain, compared to healthy subjects. $[10,16-18,21,23]$ Patients with visual aura migraine also had greater alpha peak power in their interhemispheric asymmetry in the posterior regions, unrelated to the side of the headache. $[10,24]$

3. In magneto-encephalography research in migraine patients with aura, there has been shown increased functional connectivity in the theta band in the occipital lobe area $[25,26]$

4. In migraine patients with aura, there have also been reports of mild asymmetry of the slow waves in the fronto-temporaloccipital area contralateral to the side of the visual field defects. $[10,20,21]$ 
In the literature, there has also been a case report on kaleidoscopic visual illusions in a patient using stimulant-containing diet pills. Interestingly, this patient's brother also had the same symptoms when he was abusing stimulant agents, but the symptoms stopped when he stopped using these. [11]

Whereas the brainstem trigeminal nuclei is well known to be involved in the pathophysiology of migraine without aura, there is no conclusive studies or reports to date pertaining to patients with migraine aura. In summary, resting electric and magnetic activities may help to differentiate migraine with and without aura. [10, 21, 27, 28]

\section{Discussion}

In the approach to a patient presenting with migraine with aura, it is important to get a comprehensive history and rule out possible differential diagnoses. Whilst having arrhythmia such as atrial fibrillation (AF) can predispose one to stroke and transient ischemic attacks (TIA), many older patients and those with conditions such as valvular heart disease may have both AF and migraine. Symptoms such as homonymous visual disturbance can happen in both stroke and migraine as well. [6, 18] On the other hand seeing 'colors and zig zag lines' are more likely due to migraine aura. Information in the history such as patent foramen ovale, occipital epilepsy and list of drugs patients are taking can be very useful. $[6,14,24,26]$

For the visual aura which is persistently long ( $>2$ hours) it is important to rule out infarction. This is very rare as most visual aura last less than 60 minutes. It is also useful to be aware of the definition of 'migraine aura status'; ie. experiencing 3 aura within 3 days, although this is also very rare. To rule out differentials such as occipital lobe epilepsy, performing an EEG (electro-encephalography) will help differentiate this from migraine aura. If unsure in any circumstances, it will be better to admit the patient for further investigations. [2,8, 12, 14]

\section{Conclusion}

A variety of visual aura manifestations can occur with migraine. It is useful to be aware of this spectrum. Kaleidoscopic visual aura in migraine, as described here, is unique and interesting. To date there are several postulations but not a common consolidated agreement about what causes and where the exact cascade of events brings forth migraine aura, lest to say, specifically with kaleidoscopic visual aura as in this case.

Our patient is receiving further investigations and regular follow up with the Neurologist for her condition.

\section{References}

1. Headache Classification Committee of the International Headache Society: The International Classification of Headache Disorder, $3^{\text {rd }}$ Edition. Cephalgia 2018; 38(1): 1-211

2. Welch KM. Contemporary concepts of migraine pathogenesis. Neurology 2003; 61(8 Suppl 4): S2-8

3. Schott GD. Exploring the visual hallucinations of migraine aura: The tacit contributions of illustration. Brain 2007; 130(Pt 6): $1690-1703$

4. Hansen JM, Goadsby PJ, Charles AC. Variability of clinical features in attacks of migraine with aura. Cephalgia 2016; 36(30: 216-224

5. Vianna M, Linde M, Sances G. Migraine aura symptoms: Duration, succession and temporal relationship to headache. Cephalgia 2016; 36(5): 413-421

6. Lee MJ, Lee C, Chung CS. The migraine-stroke connection. J Stroke 2016; 18(2): 146-156
7. Luedtke K, Schulte LH, May A. Visual processing in migraineurs depends on the migraine cycle. Ann Neurol 2019; 85(2): 280-283

8. Terrin A, Mainardi F, Maggioni F. The pathological spectrum behind migraine aura status. A case series. Neurol Sci 2019; 40(4): 861-864

9. Bernstein CA, Rony- Reuven N, Noseda R, Fulton AB, Huntington S, Lee AJ, Bertisch SM et al. The migraine eye: Distinct rod-driven retinal pathway response to dim light challenges and the visual cortex hyperexcitability Theory. Pain 2019; 160(3): 569-578

10. Coppola G, Di Lorenzo C, Parisi V, Lisicki M, Serrao MPierelli F. Clinical neurophysiology of migraine with aura. The $\mathrm{J}$ of Headache and Pain 2019; 20: 42.

11. Winslow H, Mickey B, Frohman EH. Sympathomimeticinduced kaleidoscopic visual illusion associated with a reversible splenium lesion. Arch Neurol 2006; 63:135-137

12. Hadjikhani N, Vincent M. Visual perception in migraine: A narrative review. Vision 2021; 5: 20.

13. Istrate $\mathrm{BM}$, Vilciu $\mathrm{C}$, Ragan C. Retinal Migraine. Rom J Opthalmol 2020; 64(20: 96-99

14. Dafer RM. What is migralepsy?

15. Wolf ME, Szabo K, Griebe M. Clinical and MRI characteristics of acute migrainous infarction. Neurology 2011; 76(22): 19111917

16. Hodak J, Fischer CL, Bassetti CLA, Schonken CJ. Episodic visual snow associated with migraine attacks. JAMA neurology 2020; 77:392-393

17. Hartl E, Gonzalez Victores JA, Remi J. Visual auras in epilepsy and migraine: An analysis of clinical characteristics. Headache 2017; 57(6): 908-916

18. Buchholz DW, Reich SG. The menagerie of migraine. Semin Neurol 1996; 16: 83-93

19. Conway BR. Color vision, color coding and cones in the cortex. Neuroscientist 2009; 15(3): 274-290

20. Coppola G, Pierelli F, Schoenen J. Is the cerebral cortex hyperexcitable or hyper-responsive in migraine? Cephalgia 2007: 27(12): 1427-1439

21. Valeriani M, Fierro B, Brighina F. Brain excitability in migraine: Excitability or inhibited inhibition? Pain 2007; 132(1-2): 219220

22. Charles A, Baca SM. Cortical spreading depression and migraine. Nat Rev 2013; 9: 637-644

23. Sand T, White LR, Hagen K, Stovner LJ. Visual evoked potential and spatial frequency in migraine: a longitudinal study. Acta Neurol Scand 2009; 189: 33-37

24. Arngrim N, Hougaard A, Ahmadi K. Heterogenous migraine aura symptoms correlate with visual cortex functional MRI responses. Ann Neurol 2017; 82: 925-939

25. Hall SD, Barnes GR, Hilebrand A. Spatio-temporal imaging of cortical desynchronization in migraine visual aura: a magnetoencephalography case study. Headache 2004; 44 204-208

26. Fong CY, Law WHC, Braithwaite JJ, Nazaheri A. Difference in early and late pattern onset visual evoked potentials between self reported migraineurs and controls. NeuroImage Clin 2020; 25: 102122

27. Martin VT, Behbehani MM. Towards a rational understanding of migraine trigger factors. Med Clin North Am 2001; 85: 911 941

28. Podoll K, Robinson D, Nicola U, Ayles D. Kaleidoscopic hallucination as visual aura symptom in migraine. Confinia Cephalalgica 2003; 12(2): 53-63 